\section{Contexte}

Le cancer du poumon est le premier cancer dans le monde en termes d'incidence et de mortalité. On estime en 2018 à près de 2,1 millions le nombre de nouveaux cas de cancer du poumon et à 1,8 million le nombre de décès [1].

Le principal facteur de risque des cancers du poumon est le tabac, qui est responsable d'au moins 8 cancers du poumon sur 10 dans les pays occidentaux. Les fumeurs ont un risque de cancer du poumon environ 10 fois plus élevé que les non-fumeurs, risque qui augmente avec la durée du tabagisme et la quantité de cigarettes fumées, la durée étant le déterminant le plus important. D'autres formes de tabagisme, comme le cigare et la pipe, ont également été associées à un risque accru de cancer du poumon bien que ce risque apparaisse cependant plus faible qu'avec la cigarette. Le tabagisme augmente le risque de tous les types histologiques de cancer du poumon. Enfin, le tabagisme passif est également associé à une augmentation du risque de cancer du poumon de 20 à $30 \%$ [2].

Si le tabagisme est le facteur de risque majeur, de nombreux autres facteurs de risque de cancer du poumon ont été identifiés. Une grande partie d'entre eux sont des substances ou situations d'exposition présentes principalement en milieu professionnel, le cancer du poumon étant d'ailleurs le plus fréquent des cancers professionnels. L'amiante en est la cause la plus connue et la plus fréquente, mais plusieurs autres expositions professionnelles sont des facteurs de risque avérés, notamment la silice cristalline, les gaz d'échappement diesel, les hydrocarbures aromatiques polycycliques (HAP), divers métaux (arsenic, cadmium, béryllium, certains composés du chrome et du nickel), les fumées de soudage et les rayonnements ionisants. Des incertitudes subsistent pour plusieurs cancérogènes pulmonaires professionnels probables, comme par exemple les bitumes ou les insecticides non arsenicaux [3]. La pollution atmosphérique et les particules fines de l'air extérieur sont également des causes reconnues de cancer du poumon [4].

\title{
Facteurs de risque professionnels et environnementaux des cancers du poumon aux Antilles françaises
}

Léilla Cabréra ${ }^{1}$, Aviane Auguste ${ }^{1}$, Léah Michineau ${ }^{1}$, Jacqueline Deloumeaux ${ }^{2}$, Clarisse Joachim ${ }^{3}$, Danièle Luce ${ }^{1}$

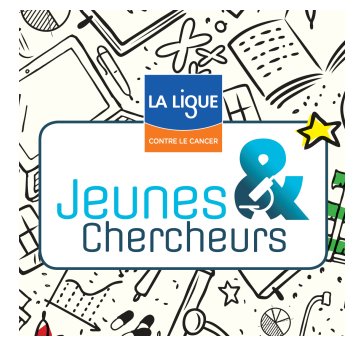

De nombreuses études suggèrent que les facteurs alimentaires contribuent également au risque de cancer du poumon. Une alimentation riche en fruits et légumes a été associée de façon répétée à une diminution de l'incidence du cancer du poumon [5]. En revanche, une consommation importante de viande rouge, de graisses saturées, ainsi que les produits à base de viande salée et fumée, augmenteraient le risque de cancer du poumon. Contrairement à ce qui est observé pour la plupart des cancers, l'obésité est associée à une diminution du risque de cancer du poumon, mais un effet de confusion résiduel lié à un moindre tabagisme ne peut être exclu. Les apparentés au premier degré de personnes atteintes de cancer du poumon ont un risque plus élevé de développer la maladie, ce qui indique une possible susceptibilité génétique. Des études pangénomiques ont mis en évidence plusieurs variants génétiques, associés cependant à de faibles augmentations de risque et qui n'expliquent qu'une partie du risque familial. Le rôle de plusieurs autres facteurs de risque a été évoqué. Les antécédents de certaines maladies pulmonaires, comme la bronchopneumopathie chronique obstructive (BPCO), l'asthme ou la tuberculose pourraient contribuer à la survenue du cancer du poumon. Des associations entre consommation d'alcool et de cannabis ont été rapportées. Le rôle potentiel de certains virus, notamment le papillomavirus humain (HPV) est discuté [6]. 
En Guadeloupe et en Martinique, les cancers du poumon sont moins fréquents qu'en France hexagonale. Les taux d'incidence standardisés sur l'âge (monde) pour 100000 sur la période 2007-2016 sont, chez les hommes, respectivement de 12,1 en Guadeloupe, 10,3 en Martinique contre 51,8 en France hexagonale; chez les femmes, ces taux sont respectivement de 4,4,6,3 et 17,9 [7].

Cette incidence plus faible s'explique principalement par une consommation de tabac plus faible [8]. En effet, la proportion de fumeurs est de $23 \%$ chez les hommes et $13 \%$ chez les femmes aux Antilles contre respectivement $32,3 \%$ et $24,3 \%$ en France hexagonale. Cette population à faible prévalence du tabagisme est donc particulièrement intéressante pour étudier d'autres facteurs de risque des cancers du poumon, au premier rang desquels figurent les expositions professionnelles et environnementales. L'implication d'autres facteurs de risque que le tabac est également suggérée par une étude descriptive portant sur les cancers du poumon en Guadeloupe [9], qui a mis en évidence une proportion élevée de cas non-fumeurs.

La population antillaise présente des particularités en matière de risques professionnels, avec des activités spécifiques comme la culture de la banane ou l'industrie de la canne à sucre. Des excès de cancer du poumon ont été rapportés au Costa Rica chez les travailleurs de plantations de banane [10]. Des risques élevés de cancer du poumon ont également été mis en évidence dans la culture de la canne à sucre en Inde [11], pouvant être dus à la présence de fibres de silice amorphe biogéniques dans les feuilles. Ces fibres ont des caractéristiques proches de celles de l'amiante, et peuvent également se transformer en silice cristalline à haute température, pendant la fabrication du sucre ou lors du brûlage des cannes avant ou après la coupe. Le brûlage des cannes peut entraîner par ailleurs des émissions de HAP, pouvant toucher aussi les habitations environnantes [12]. Le nombre d'études est cependant limité et ces résultats demandent à être répliqués dans d'autres populations.

Les Antilles sont également caractérisées par une utilisation importante de pesticides. Des associations entre le risque de cancer du poumon et plusieurs familles de pesticides ou substances actives utilisées aux Antilles ont été suggérées, notamment avec les carbamates, des organophosphorés comme le diazinon, et des organochlorés comme la dieldrine et le lindane $[13,14]$. Des épandages aériens de fongicides ont également été effectués sur les bananeraies depuis la fin des années 1950 pour lutter contre la cercosporiose ${ }^{1}$.

$\varepsilon n$ dehors de ces expositions spécifiques, les particularités du tissu économique aux Antilles permettent d'être dans une situation favorable pour étudier le rôle d'expositions professionnelles. La plupart des activités sont représentées, mais la forte proportion de très petites entreprises (plus de $90 \%$ ) et la fréquence élevée de l'emploi informel sont associées à des conditions de travail plus péjoratives qu'en France hexagonale.

\footnotetext{
${ }^{1}$ La cercosporiose est un ensemble de maladies fongiques foliaires dues aux champignons du genre des Cercospora. Celle du bananier a été reclassée dans le genre Mycosphaerella car due en fait au champignon Mycosphaerella musicola.
}

Le rôle des expositions professionnelles et environnementales dans l'étiologie des cancers du poumon reste donc largement méconnu aux Antilles. Aussi, l'objectif principal est d'examiner le rôle de ces facteurs, et, plus généralement, de clarifier le rôle et l'impact de différents facteurs de risque sur le risque de cancer du poumon. Plus spécifiquement, il s'agit pour nous (1) d'étudier les associations entre expositions professionnelles et risque de cancer du poumon, (2) d'étudier le rôle éventuel d'expositions résidentielles, (3) d'étudier dans cette population d'autres facteurs de risque connus ou suspectés et les interactions entre ces facteurs et les expositions professionnelles et environnementales, et enfin (4) d'évaluer la proportion de cas de cancer du poumon attribuables aux différents facteurs de risque dans ces régions.

\section{Méthodes}

Ce travail est fondé sur les données d'une étude castémoins en population générale, menée en Guadeloupe et en Martinique entre 2013 et 2016 . Cette étude est une extension aux Antilles de l'étude Icare, une large étude cas-témoins sur les cancers respiratoires conduite en France hexagonale [15], et a utilisé le même protocole et questionnaire, avec quelques adaptations au contexte local.

Les cas ont été identifiés en collaboration avec les registres des cancers des deux régions. Les cas éligibles sont tous les patients atteints d'une tumeur primitive de la trachée, des bronches et du poumon (classification internationale des maladies, $10^{e}$ révision, codes C33-C34) nouvellement diagnostiquée entre le $1^{\text {er }}$ avril 2013 et le 31 décembre 2016, résidant en Guadeloupe ou en Martinique et âgés d'au plus 75 ans au moment du diagnostic. Tous les types histologiques ont été inclus. Le groupe témoin est un échantillon aléatoire de la population générale de la zone d'étude. Le recrutement a été réalisé par une procédure d'appels téléphoniques aléatoires, incluant les téléphones cellulaires. Le recrutement a été stratifié de façon à obtenir une répartition des témoins par âge, sexe et département comparable à celle des cas de cancers. Une stratification supplémentaire a été utilisée pour obtenir une répartition par catégorie socio-professionnelle comparable à celle de la population (obtenue à partir des données du recensement), afin de contrôler les biais de sélection potentiels résultant de taux de participation différentiels selon la catégorie socio-professionnelle. Les cas et les témoins ont été interrogés par des enquêteurs spécialement formés, avec un questionnaire standardisé portant notamment sur les caractéristiques 


\begin{tabular}{|c|c|c|c|c|}
\hline & \multicolumn{2}{|c|}{ Cas $(n=147)$} & \multicolumn{2}{|c|}{$\begin{array}{l}\text { Témoins } \\
(n=405)\end{array}$} \\
\hline & $n$ & $\%$ & $\mathrm{n}$ & $\%$ \\
\hline \multicolumn{5}{|l|}{ Sexe } \\
\hline Homme & 90 & $61,2 \%$ & 306 & $75,6 \%$ \\
\hline Femme & 57 & $38,8 \%$ & 99 & $24,4 \%$ \\
\hline \multicolumn{5}{|l|}{ Âge (années) } \\
\hline$<45$ & 4 & $2,7 \%$ & 62 & $15,3 \%$ \\
\hline $45-54$ & 25 & $17,0 \%$ & 107 & $26,4 \%$ \\
\hline $55-64$ & 54 & $36,7 \%$ & 129 & $31,9 \%$ \\
\hline$>65$ & 64 & $43,5 \%$ & 107 & $26,4 \%$ \\
\hline \multicolumn{5}{|l|}{ Tabac } \\
\hline Non-fumeurs ${ }^{a}$ & 60 & $40,8 \%$ & 266 & $65,7 \%$ \\
\hline Fumeurs actuels & 54 & $36,7 \%$ & 53 & $13,1 \%$ \\
\hline$\varepsilon x$-fumeurs ${ }^{b}$ & 33 & $22,5 \%$ & 86 & $21,2 \%$ \\
\hline \multicolumn{5}{|l|}{ Type histologique ${ }^{c}$} \\
\hline Carcinomes épidermoïdes & 28 & $19,0 \%$ & & \\
\hline Adénocarcinomes & 91 & $62,0 \%$ & & \\
\hline Autres types histologiques & 28 & $19,0 \%$ & & \\
\hline
\end{tabular}

Tableau I. Principales caractéristiques des cas et des témoins. aNon-fumeurs : personnes ayant consommé moins de 100 cigarettes au cours de leur vie. ${ }^{b} \varepsilon_{x}$ fumeurs : personnes ayant arrêté de fumer depuis au moins deux ans. 'Les codes morphologie de la CIM-0-3 ont été regroupés de la façon suivante : épidermoïdes : 8050-8078, 8083, 8084 ; adénocarcinomes: 8140, 8211, 8230, 8231, $8250-8260,8323,8480-8490,8550,8551,8570-8574,8576$.

socio-démographiques, les consommations de tabac, l'histoire résidentielle complète, l'histoire professionnelle détaillée, avec une description de chaque emploi occupé, ainsi que des questionnaires spécifiques pour certaines tâches ou professions. Les autres informations recueillies concernent les caractéristiques anthropométriques, les facteurs hormonaux chez les femmes, les antécédents de cancers et de maladies diverses, le cancer chez les apparentés au premier degré, les consommations d'alcool, de cannabis et l'alimentation.

Pour chaque sujet, tous les emplois exercés pendant la vie active (profession et branche d'activité) ont été codés, en utilisant, pour la profession, la classification internationale type des professions (CITP) du bureau international du travail (BIT) de 1968, et pour la branche d'activité la nomenclature d'activités française (NAF) de I'Insee (institut national de la statistique et des études économiques) de 2000. Les expositions professionnelles seront évaluées à partir des réponses au questionnaire et/ou de matrices emplois-expositions, avec la collaboration d'experts locaux en santé au travail (ingénieur de prévention, médecins du travail). Les différentes adresses au cours de la vie sont en cours de géocodage.

Les données sont analysées par les méthodes classiquement utilisées pour les études cas-témoins. Des modèles de régression logistique non conditionnelle sont utilisés pour estimer les odds-ratios $(O R)^{2}$ ajustés et leurs intervalles de confiance à $95 \%$ (IC $95 \%$ ), et pour évaluer les interactions.

\section{Résultats préliminaires}

Au cours de la période d'étude, 237 cas potentiellement éligibles ont été identifiés. Parmi ceux-ci, 13 sont décédés avant d'avoir pu être contactés. Parmi les 224 cas contactés, 55 ont refusé de participer, 169 (75\%) ont été interrogés. Après vérification avec les données des registres, 22 cas ont été exclus a posteriori car ils ne remplissaient pas les critères d'inclusion (notamment, récidives ou date de premier diagnostic hors période). Au total, 147 cas ont été inclus dans l'étude. Sur les 497 témoins éligibles recrutés par l'institut de sondage, 16 n'ont pas pu être contactés, 76 ont refusé de participer et 405 (81\%) ont été interrogés.

Les principales caractéristiques des cas et des témoins sont présentées dans le Tableau I.

La proportion de fumeurs est, comme attendu, plus élevée chez les cas de cancers que chez les témoins. La proportion de cas n'ayant jamais fumé est cependant particulièrement élevée, plus de $40 \%$. Cette proportion élevée de cas non-fumeurs suggère le rôle d'autres facteurs de risque.

L'examen de la répartition des cas en fonction du type histologique met en évidence une forte proportion d'adénocarcinomes, plus élevée qu'en France hexagonale. Cette prédominance des adénocarcinomes est retrouvée dans d'autres régions caribéennes.

Les premières analyses, qui doivent être approfondies, ont déjà permis de mettre en évidence des risques élevés de cancer du poumon dans certaines professions (Figure 1) ou secteurs d'activité (Figure 2). Les résultats suggèrent un rôle d'expositions liées au travail de la canne à sucre, avec des augmentations significatives de risque chez les ouvriers agricoles de la canne à sucre, dans le secteur des cultures industrielles, et, dans une moindre mesure, dans la production de rhum. Des risques élevés de cancer du poumon ont également été observés chez les autres ouvriers agricoles, les

${ }^{2}$ L'odds ratio est une mesure statistique exprimant le degré de dépendance entre des variables aléatoires qualitatives. 

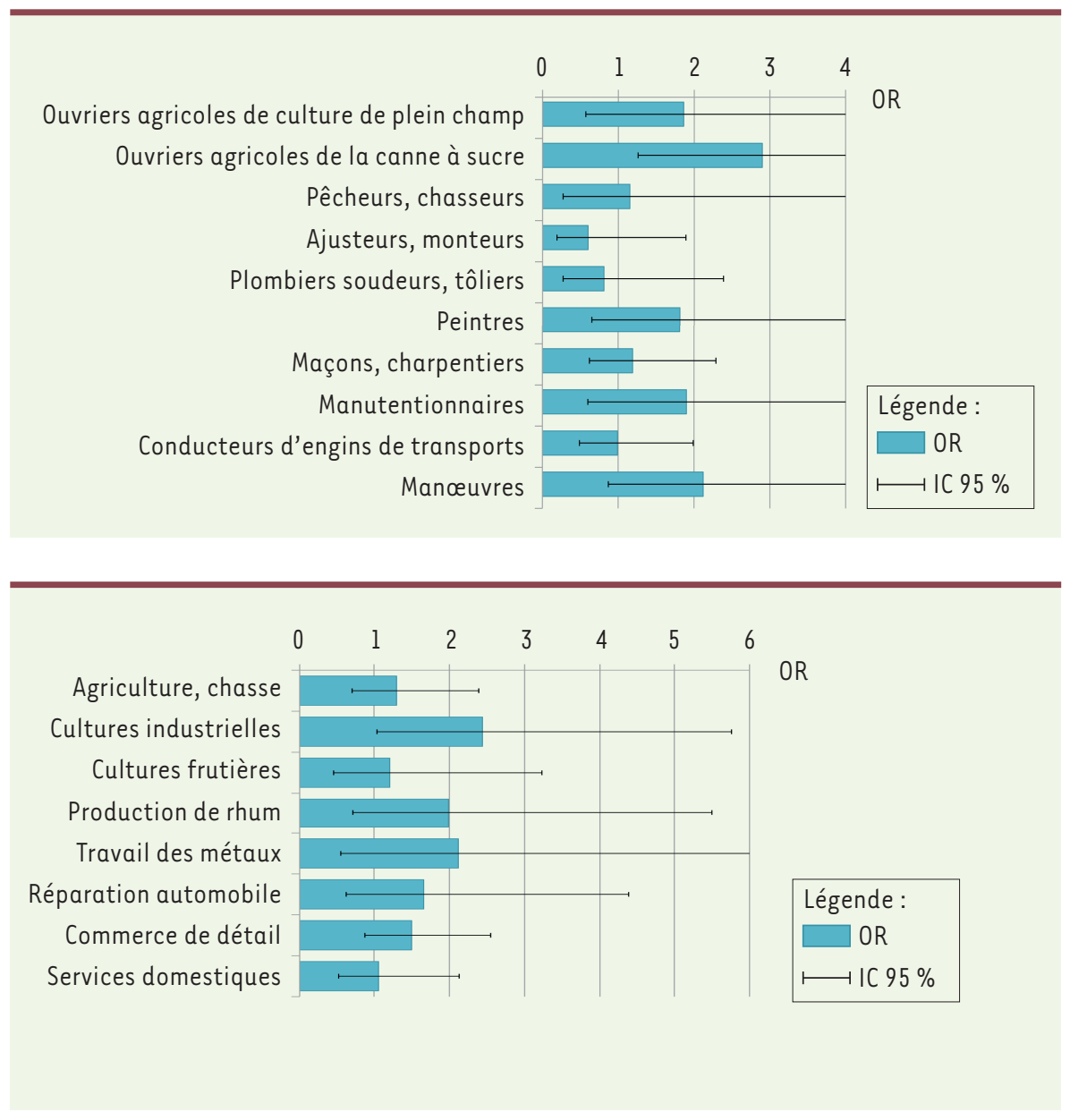

Figure 1. Association entre cancer du poumon et professions. Estimation des odds-ratio (OR) et de leur intervalle de confiance à $95 \%$ (IC $95 \%$ ) par régression logistique; ajustés sur âge, sexe, région et consommation de tabac.

Figure 2. Association entre cancer du poumon et secteurs d'activité. Estimation des odds-ratio (OR) et de leur intervalle de confiance à $95 \%$ (IC $95 \%$ ) par régression logistique; ajustés sur âge, sexe, région et consommation de tabac. manutentionnaires, les manœuvres, les peintres, ainsi que chez les personnes travaillant les métaux et dans la réparation automobile.

\section{Perspectives et retombées attendues}

II s'agit de la première étude épidémiologique sur les cancers du poumon dans les Caraïbes. L'étude permettra d'évaluer le rôle d'expositions professionnelles qui ne peuvent être étudiées que dans quelques populations, comme les expositions dans la culture de la banane, ainsi que dans la culture et l'industrie de la canne à sucre. L'utilisation importante de pesticides dans ces régions permet également d'être dans une situation favorable pour étudier leur rôle dans la survenue de cancers du poumon, évoqué dans plusieurs études. L'étude des facteurs de risque professionnels va se poursuivre par une analyse en fonction des tâches et des substances, à partir des informations détaillées recueillies dans les questionnaires. Le recueil complet de l'histoire résidentielle permettra d'évaluer l'association entre risque de cancer du poumon et habitat à proximité d'activités potentiellement polluantes, notamment le brûlage de champs de canne à sucre et l'épandage aérien de bananeraies; la proximité d'autres activités, centrales thermiques au charbon ou décharges par exemple, pourra également être étudiée. Les autres facteurs de risque connus ou suspectés (tabac, cannabis, alcool, alimentation, antécédents familiaux, antécédents de maladies respiratoires...) seront également étudiés et pris en compte dans l'analyse des facteurs professionnels et environnementaux, et les éventuelles interactions entre ces facteurs seront évaluées.

L'étude permettra d'évaluer au niveau régional l'impact des différents facteurs de risque sur le risque de cancer du poumon, et ainsi de mieux orienter les actions de prévention. En particulier, une estimation du nombre de cas de cancer du poumon attribuables aux expositions professionnelles dans ces régions peut avoir des implications importantes à la fois pour la prévention et la prise en charge médico-administrative de ces cancers au titre des maladies professionnelles. $\diamond$

Occupational and environmental risk factors for lung cancer in the French West Indies

\section{LIENS D'INTÉRÊT}

Les auteurs déclarent n'avoir aucun lien d'intérêt concernant les données publiées dans cet article. 


\section{RÉFÉRENCES}

1. Ferlay J, Colombet M, Soerjomataram I, et al. Estimating the global cancer incidence and mortality in 2018: GLOBOCAN sources and methods. Int J Cancer 2019 ; 144 : 1941-53.

2. IARC. Tobacco smoke and involuntary smoking. IARC Monogr Eval Carcinog Risks Hum 2004 ; 83 $1-1438$.

3. Delva F, Andujar P, Lacourt A, et al. Occupational risk factors for lung cancer. Rev Mal Respir $2016 ; 33: 444-59$

4. Loomis D, Grosse $Y$, Lauby-Secretan B, et al. The carcinogenicity of outdoor air pollution. Lancet Oncol $2013 ; 14: 1262-3$

5. Vieira AR, Abar L, Vingeliene $S$, et al. Fruits, vegetables and lung cancer risk: a systematic review and meta-analysis. Ann Oncol 2016 ; $27: 81-96$.

6. Malhotra J, Malvezzi M, Negri $\varepsilon$, et al. Risk factors for lung cancer worldwide. Eur Respir J 2016; $48: 889-902$.

7. Santé Publique France. Estimations régionales et départementales de l'incidence et de la mortalité par cancer en France, 2007-2016. https://www.santepubliquefrance.fr/maladies-ettraumatismes/cancers/articles/estimations-regionales-et-departementales-de-l-incidence-etde-la-mortalite-par-cancer-en-france-2007-2016

8. Auguste A, Dugas J, Menvielle G, et al. Social distribution of tobacco smoking, alcohol drinking and obesity in the French West Indies. BMC Public Health 2019; 19 : 1424.

9. Cadelis $\mathrm{G}$, Kaddah $\mathrm{S}$, Bhakkan $\mathrm{B}$, et al. Epidemiology and incidence of primary lung cancer in a region with low tobacco consumption: Guadeloupe (French West Indies). Data from the cancer registry 2008-2009. Rev Mal Respir 2013 ; 30 : 537-48.
10. Hofmann J, Guardado J, Keifer M, et al. Mortality among a cohort of banana plantation workers in Costa Rica. Int J Occup Environ Health 2006; 12 : 321-8.

11. Amre DK, Infante-Rivard C, Dufresne A, et al. Case-control study of lung cancer among sugar cane farmers in India. Occup Environ Med 1999 ; 56 : 548-52.

12. Cristale J, Silva FS, Zocolo GJ, et al. Influence of sugarcane burning on indoor/ outdoor PAH air pollution in Brazil. Environ Pollut 2012; 169 : 210-16.

13. Bonner MR, Freeman LEB, Hoppin JA, et al. Occupational exposure to pesticides and the incidence of lung cancer in the agricultural health study. Environ Health Perspect 2017 ; 125 : 544-51.

14. Alavanja MCR, Dosemeci M, Samanic C, et al. Pesticides and lung cancer ris in the agricultural health study cohort. Am J Epidemiol 2004 ; 160 : 876-85.

15. Luce D, Stücker I, ICARE Study Group. Investigation of occupational and environmental causes of respiratory cancers (ICARE): a multicenter, population-based case-control study in France. BMC Public Health 2011; $11 \cdot 928$

TIRÉS À PART

L. Cabréra

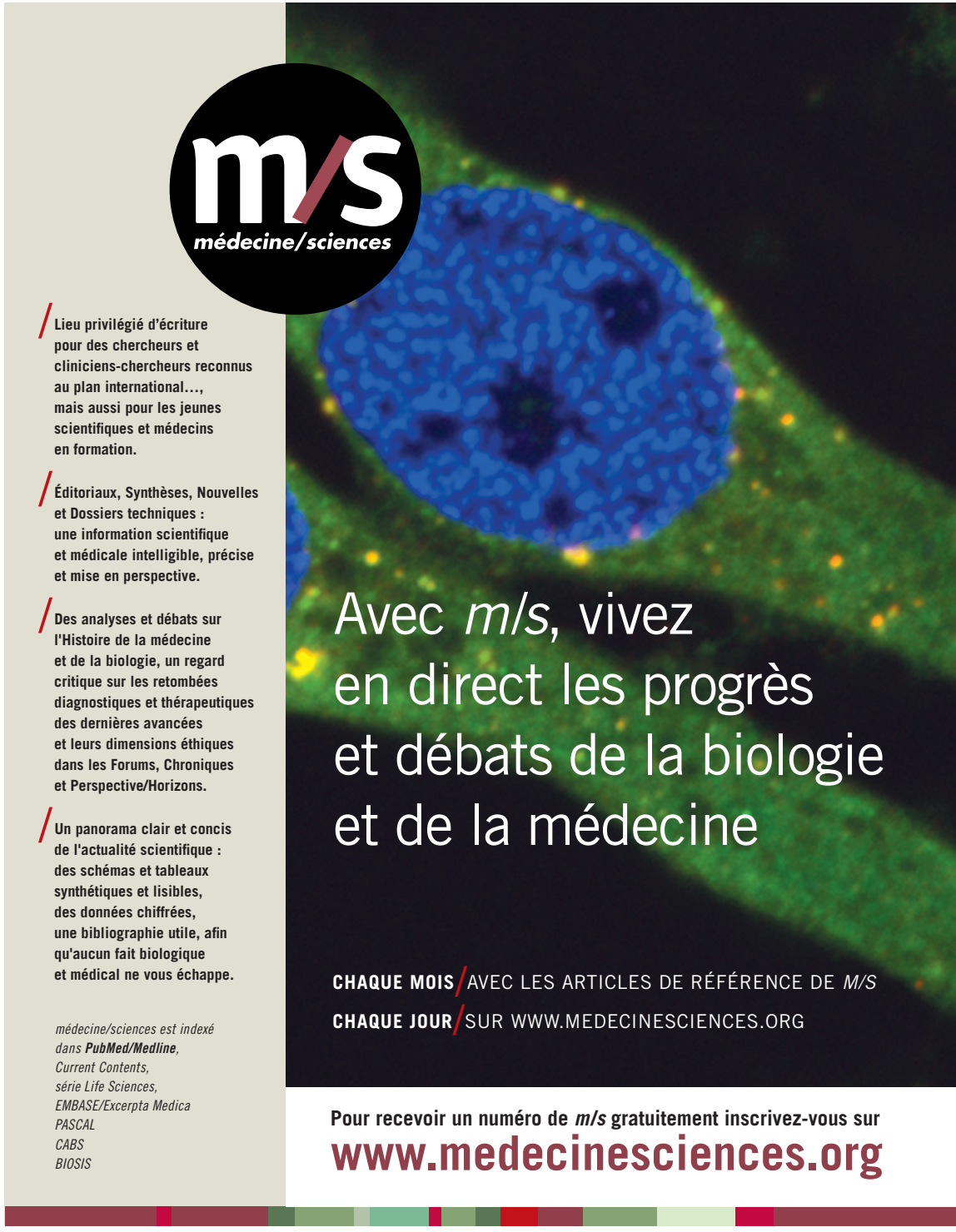

